\title{
Technologieadoptionsstrategien von Medienunternehmen. Schnelligkeit, Zeitpunkt und Planungshorizonte von Investitionen und Akquisitionen in Augmented und Virtual Reality-Technologien
}

\author{
Magdalena Ciepluch • Uwe Eisenbeis
}

Eingegangen: 28. Juli 2021 / Angenommen: 17. November 2021 / Online publiziert: 20. Januar 2022

(C) Der/die Autor(en) 2022

Zusammenfassung Augmented und insbesondere Virtual Reality-Technologien und -Anwendungen existieren zwar bereits seit einigen Jahrzehnten, durchleben jedoch seit einigen Jahren (erneut) einen Aufschwung, getrieben durch technologische Entwicklungen. Aus diesen Entwicklungen ergeben sich in diversen Branchen, insbesondere aber in der Medienbranche, sowohl vielerlei Chancen als auch Herausforderungen. Vor diesem Hintergrund stellt sich die Frage, wie sich Unternehmen der Medienbranche auf diese neuen Technologien einstellen bzw. eingestellt haben im Sinne einer strategischen Vorbereitung auf die Zukunft bzw. hinsichtlich ihrer Technologieadoptionsstrategien. In diesem Artikel wird untersucht, wie früh oder spät bzw. zu welchem Zeitpunkt AR- und VR-Investitionen stattfinden und welchen Planungshorizont (Dauer vom Zeitpunkt der Investition bis zum Erreichen des Produktionslevels) Medienunternehmen in ihren AR- und VR-Investitionen verfolgen. Die Ergebnisse zeigen, dass sich diejenigen Medienunternehmen, die in AR und VR investieren, in zwei große Gruppen aufteilen lassen: Wenn investiert wird, geschieht dies entweder relativ früh (nach 2 bis 4 Jahren) oder relativ spät (erst nach 12 bis 14 Jahren) nach Aufkommen der Technologie. Die beiden Gruppen können als Early Majority und Late Majority verstanden werden. Gemeinsam ist allen Medienkonzernen, dass ihre Investitionen einen mittelfristigen Planungshorizont von 5 bis 10 Jahren aufweisen, der bei AR sogar tendenziell länger ist als bei VR. Medienunternehmen sind sich dem langfristigen (Markt-)Potenzial beider Technologien offenbar bewusst, investieren aber nur sehr zaghaft in diese.

\footnotetext{
Magdalena Ciepluch $(\bowtie) \cdot$ Uwe Eisenbeis

Fakultät Electronic Media, Studiengang Medienwirtschaft, Hochschule der Medien,

Nobelstraße 10, 70569 Stuttgart, Deutschland

E-Mail: ciepluch@hdm-stuttgart.de

Uwe Eisenbeis

E-Mail: eisenbeis@hdm-stuttgart.de
} 
Schlüsselwörter Virtual Reality · Augmented Reality ·

Technologieadoptionsstrategien · Technologieinvestitionen · Medienkonzerne •

Timing Strategy $\cdot$ Planungshorizont

\title{
Technology adoption strategies of media companies. Speed, timing and planning horizons of investments and acquisitions in augmented and virtual reality technologies
}

\begin{abstract}
Augmented and especially Virtual Reality technologies and applications have existed for several decades, but have been experiencing a (renewed) upswing in recent years, driven by technological developments. These developments result in a variety of opportunities as well as challenges in various industries, but especially in the media industry. Against this background, the question arises as to how companies in the media industry are adopting or have adopted to these new technologies - in the sense of strategic preparation for the future or their technology adoption strategies. This article examines how early or late, or at what point in time, $\mathrm{AR}$ and VR investments take place and what planning horizon (duration from the time of investment to reaching production level) media companies follow in their $\mathrm{AR}$ and VR investments. The results show that those media companies investing in AR and VR fall into two broad groups: When investments are made, they occur either relatively early (after 2 to 4 years) or relatively late (only after 12 to 14 years) after the technology has emerged. These two groups can be understood as Early Majority and Late Majority. What all media groups have in common is that their investments have a medium-term planning horizon of 5 to 10 years, which even tends to be longer for AR than for VR. Media companies are obviously aware of the long-term (market) potential of both technologies, but are very hesitant to invest in them.
\end{abstract}

Keywords Virtual Reality · Augmented Reality • Technology Adoption Strategy • Technology Investments · Media Companies · Timing Strategy · Planning Horizon

\section{Einleitung und Zielsetzung}

Augmented Reality- und insbesondere Virtual Reality-Technologien und -Anwendungen existieren zwar bereits seit einigen Jahrzehnten, durchleben jedoch seit einigen Jahren (erneut) einen Aufschwung, getrieben durch technologische Entwicklung und die damit zusammenhängende höhere Leistungsfähigkeit (van Gisbergen et al. 2019) der Technologien und Systeme. Aus diesen Entwicklungen ergeben sich in diversen Branchen, insbesondere aber in der Medienbranche, sowohl vielerlei Chancen als auch Herausforderungen. Für Medienunternehmen stellen Technologien eine Differenzierungsmöglichkeit sowie die Möglichkeit des Herausbildens von Wettbewerbsvorteilen in den Bereichen ihrer Geschäftsmodelle und Wertschöpfungsketten dar (Godefroid und Kühnle 2018) und haben somit das Potenzial, den Markt spürbar zu verändern (Mütterlein et al. 2017). Zudem kann inzwischen angenommen werden, dass Augmented und Virtual Reality-Anwendungen auch für den Massenmarkt 
tauglich sind und dort in Zukunft substanziell nachgefragt werden (IDC 2020a, b; TrendForce 2020; Grand View Research 2021).

Vor diesem Hintergrund stellt sich die Frage, wie sich Unternehmen der Medienbranche auf diese neuen Technologien einstellen bzw. eingestellt haben - im Sinne einer strategischen Vorbereitung auf die Zukunft (um wettbewerbsfähig zu sein bzw. zu bleiben). Das „Sich-Einstellen“ auf (neue) Technologien sowie die entsprechende Vorbereitung der Unternehmen auf die Zukunft spiegelt sich im sogenannten Technologieadoptionsverhalten bzw. in den sogenannten Technologieadoptionsstrategien wider (Jensen 1982, 1992; McCardle 1985; Stenbacka und Tombak 1994; Hoppe 2002). Im konkreten Fall zeigen die Technologieadoptionsstrategien unter anderem, wie Medienkonzerne auf die Technologien Augmented und Virtual Reality reagieren (Zabel und Telkmann 2020).

In der managementorientierten Betriebswirtschaftslehre beinhaltet eine strategische Vorbereitung auf die Zukunft insbesondere auch ,Investitionen in (diese) Technologien“, wobei Investitionen sowohl intern (z.B. zum Aufbau entsprechender Innovationslabs, Forschungs- und Entwicklungsbemühungen, Weiterbildungsmaßnahmen etc.) als auch extern (z.B. Recruiting neuer Mitarbeiter mit entsprechenden Kompetenzen, Beteiligungen an und Akquisitionen von Startups und Unternehmen aus dem entsprechenden Technologiebereich) verstanden werden können (Hess 2019). Im hier vorliegenden Fall wird das strategische Verhalten von Medienkonzernen durch die Analyse des Investitionsverhaltens (Investitionsbeteiligungen und Akquisitionen) dieser Medienkonzerne in Startups und Technologieunternehmen aus den Bereichen Augmented Reality und Virtual Reality analysiert.

Es wird zum einen untersucht, zu welchem Zeitpunkt Medienkonzerne reagiert haben (Frühzeitigkeit der Technologieninvestitionen) und wie vorausschauend sie demnach geplant haben. Zum anderen wird untersucht, wie weit in die Zukunft (bis zur erwarteten Wirtschaftlichkeit der Technologie) die Investitionen und Akquisitionen der Konzerne gerichtet sind (Planungshorizont der Technologieinvestitionen).

Insofern sollen in dem Beitrag insbesondere die zwei folgenden Fragen beantwortet werden:

1. Wie früh (oder spät) und zu welchen Zeitpunkten investieren Medienkonzerne in Augmented und Virtual Reality-Technologien und -Anwendungen?

2. Wie weit in die Zukunft planen Medienkonzerne bei den Investitionen in Augmented und Virtual Reality-Technologien und -Anwendungen?

Zur Beantwortung der Fragen werden die Technologieinvestitionen (Beteiligungsinvestitionen und Akquisitionen in Startups und Unternehmen) der weltweit 100 größten Medienkonzerne (Institut für Medien- und Kommunikationspolitik 2020) von 2000 bis 2019 untersucht. Von den insgesamt 3002 analysierten Technologieinvestitionen (davon 2550 Investitionen im Sinne von Beteiligungen und 452 Akquisitionen) sind 178 Technologieinvestitionen den Bereichen Virtual Reality (im Folgenden als VR abgekürzt) und Augmented Reality (im Folgenden als AR abgekürzt) zuzuordnen. 


\section{Theoretischer Hintergrund und Forschungsgegenstand}

Unternehmen sind in der Regel nicht automatisch auf ein sich veränderndes und zunehmend dynamisches Umfeld vorbereitet (Scholz 2000). Die wichtigste Herausforderung für die Managementpraxis ist die Sicherstellung einer strategischen Stimmigkeit zwischen der Organisation und ihrem Umfeld (Ghoshal und Nohria 1993; Scholz 2000). Das Ziel strategischer Planung ist die erfolgreiche Positionierung und das langfristige Überleben am Markt (Nötzold 1994; Müller-Stewens und Lechner 2005; Deimel 2008). Vor dem Hintergrund dynamischer Branchen, sich schnell verändernder Marktbedingungen und technologischer Entwicklung wurde die Herausforderung, Wettbewerbsvorteile durch Anpassung und Transformation von Geschäftsmodellen zu erlangen, umfassend diskutiert (Amit und Zott 2012; CasadesusMasanell und Zhu 2013): Untersuchungen haben gezeigt, dass neue Technologien und technologische Innovationen eine Anpassung der Geschäftsmodelle erfordern (Chesbrough 2010; Teece 2010; Zott et al. 2011). Ein nach wie vor viel diskutiertes Thema ist in diesem Zusammenhang die sogenannte digitale Transformation (AndalAncion et al. 2003; Berman 2012; Hess et al. 2016), eine notwendige Transformation des Geschäftsmodells der Unternehmen und Branchen, getrieben von digitalen Technologien. Die zunehmende Bedeutung von AR- und VR-Technologien und -Anwendungen sowie deren sinkende Einstiegshürden für diverse Wirtschaftszweige beeinflussen in Konsequenz das Streben nach Innovationen, um wettbewerbsfähig zu bleiben.

VR ermöglicht es virtuelle, computergenerierte Welten vollständig über sogenannte head mounted displays (HMD) - auf dem Kopf zu tragende Ausgabegeräte wahrzunehmen (Mütterlein et al. 2017; Wohlgenannt et al. 2020). „Virtual Environment/Virtual Worlds“ erschien als Technologie bereits 2007 als Vorläufer der VR-Technologie auf dem Gartner Hype Cycle of Emerging Technologies (Gartner, Inc. 2007); von 2013 bis 2017 ist der Begriff „,VR-Technologie“ auf dem Gartner Hype Cycle zu finden (Gartner, Inc. 2013, 2014, 2015, 2016, 2017). AR-Technologien und -Anwendungen befinden sich auf dem Realitäts-Virtualitäts-Kontinuum auf einer anderen Ebene als VR-Technologien (Milgram et al. 1995). AR beschreibt eine computergestützte Erweiterung der wahrgenommenen Realität. Hierbei wird die reale Umgebung des Nutzers durch virtuelle Aspekte wie Grafiken oder virtuelle Objekte ergänzt und entweder über ein HMD oder andere Endgeräte wie ein Smartphone ausgespielt (Azuma 1997; Mekni und Lemieux 2014). Auf dem Gartner Hype Cycle of Emerging Technologies ist AR erstmalig 2004 aufgeführt (Gartner, Inc. 2004).

Die Medienindustrie gehört zu den Branchen, die am meisten von neuen Technologien beeinflusst werden (Chan-Olmsted 2006). Insbesondere VR und AR üben enormen Einfluss auf die Medienbranche aus (IABM 2018; Bitkom e. V. 2019; PwC 2020). Goldman Sachs (2016) identifizieren mehrere Anwendungsbereiche für AR und VR - unter anderem medienspezifische, wie Videogames, Live Events und Video Entertainment. Besonders die Bereiche Unterhaltung und Entertainment profitieren von AR- und VR-Anwendungen, denn laut dem German Entertainment and Media Outlook 2020-2024 geht man von einer zunehmenden Verbreitung von tragbarer, autonomer VR-Hardware aus (PwC 2020). In Deutschland konnte mit dem 
Verkauf von VR-Anwendungen 2019 ein Umsatz von 66 Mio. $€$ generiert werden, was einem Wachstum zum Vorjahr von 38,2\% entspricht (PwC 2020). Dabei steuern insbesondere VR-Games einen maßgeblichen Teil zum Wachstum bei.

\subsection{Schnelligkeit und Zeitpunkt der Technologieadoption}

Die Schnelligkeit und der Zeitpunkt der Technologieadoptionen werden hier als Elemente der strategischen Planung verstanden. Zum einen geht es um den Investitionszeitpunkt im Vergleich zur Konkurrenz und in Bezug auf das Auftauchen der Technologie. Zum anderen geht es um den tatsächlichen Zeitpunkt einer Investition oder Akquisition. Die strategische Entscheidung über diesen Adoptionszeitpunkt hängt mit dem Wissen und der Einstellung zur Unternehmensumwelt zusammen.

Bereits in den 1980er-Jahren versuchte man den optimalen Adoptionszeitpunkt neuer Technologien zu ermitteln. Jensen (1982) entwickelt ein entscheidungstheoretisches Modell, in dem eine Firma mit einer neuen Technologie konfrontiert wird und entscheiden muss, ob und wann sie diese einführt. Der Adoptionszeitpunkt unter Unsicherheit der Wirtschaftlichkeit einer Technologie nach Jensen wird auf verschiedene Weise erweitert und verallgemeinert (McCardle 1985; Bhattacharya et al. 1986). McCardle (1985) zeigt auf, dass die Technologieadoptionsentscheidung eines Unternehmens von der Rentabilitätseinschätzung der Technologie abhängig ist, wobei sich Unternehmen an zwei Schwellenwerten orientieren: Wenn die Schätzung der Rentabilität der Technologie hoch genug ist, wird diese adaptiert, während die neue Technologie nicht übernommen wird, wenn der untere Rentabilitätswert unterschritten wird. Bis zur Rentabilitätseinschätzung wartet ein Unternehmen und sammelt Informationen über die neue Technologie (McCardle 1985). Bhattacharya et al. (1986) betrachtet ein entscheidungstheoretisches Modell, in dem ein einzelnes Unternehmen zu jedem Zeitpunkt drei Optionen hat: Es kann eine neue Technologie mit unsicherer Rentabilität annehmen, es kann sie ablehnen oder es kann warten und weitere Informationen über ihren Wert sammeln. Im Gegensatz zu Jensen (1982) gehen Balcer und Lippman (1984) davon aus, dass der Wert der aktuellen Technologie mit Sicherheit bekannt ist, aber dass das Unternehmen mit der Ungewissheit über das Eintreffen einer besseren Version konfrontiert ist. Ihr Modell zeigt, dass die Ankündigung einer neuen Entdeckung zu einer Verzögerung bei der Übernahme der aktuellen Technologie führen kann (Balcer und Lippman 1984).

Neben der Analyse des Adoptionszeitpunktes unter Unsicherheit findet sich einige Literatur zum Adoptionszeitpunkt neuer Technologien unter Rivalität bzw. im Wettbewerb. So kann unterschieden werden zwischen First Mover, deren Aktionen als innovativ verstanden werden, Second Mover, die auf Aktionen eines First MoverKonkurrenten reagieren, und Late Mover, die in abwartender Position verharren sowie Markt und Konkurrenten beobachten, um für spätere Reaktionen gut vorbereitet zu sein (Lieberman und Montgomery 1988; Hitt et al. 1995). Es gibt eine Vielzahl von Forschungsarbeiten zu den First Mover-Vorteilen (z.B. Vorsprung gegenüber Wettbewerben) und/oder Second Mover-Vorteilen (z.B. Vermeidung von Fehlern des First Movers) beim Timing der Einführung bzw. Adoption neuer Technologien, die auf theoretischen Modellen und insbesondere spieltheoretischen Modellen basieren (Gilbert und Birnbaum-More 1996; Hoppe 2002; Klingebiel und Joseph 2016). 
Ein zentraler Ansatz für Early Mover-Vorteile ist der spieltheoretische Ansatz von Reinganum (1981). Er stellt fest, dass die frühere Adoption neuer Technologien einer Firma einen negativen Einfluss auf den Gewinn eines Konkurrenten bei der späteren Adoption hat (Reinganum 1981). Fudenberg und Tirole (1985) gehen jedoch von der Annahme aus, dass Unternehmen auf die Aktionen ihrer Konkurrenten reagieren können und hierdurch jeglicher First Mover-Vorteil durch den Wettlauf um den ersten Platz zunichtegemacht wird (Fudenberg und Tirole 1985). Das Modell, bei dem ein Unternehmen einen Anreiz hat, die Einführung von Technologien zu verzögern, weil es im Laufe der Zeit Informationen sammeln kann und so möglicherweise die Einführung unrentabler Technologien vermeiden kann, unterstützt die Second Mover-Vorteile (Jensen 1982; McCardle 1985; Dutta et al. 1995; Hoppe und Lehmann-Grube 2001; Hoppe 2002; Doraszelski 2004).

Außerdem kann der Adoptionszeitpunkt anhand der Entscheidungsmuster von Unternehmen eingeordnet werden. Entsprechend der sogenannten Miles-Snow-Typologie (Miles und Snow 1978) werden Unternehmen in ein Modell vier strategischer Unternehmenstypen eingeordnet, wobei diese auch einen Bezug zur Schnelligkeit bzw. Frühzeitigkeit aufweisen. Der Prospector ist ständig auf der Suche nach neuen Produkten und Marktchancen, führt als Pionier oft neue Produkte oder Dienstleistungen ein und ist technologisch innovativ. Das Hauptziel des Defender ist es, Stabilität innerhalb seines Marktfeldes zu schaffen. Daher ist er nicht an der Anpassung von Technologie, Struktur oder Prozessen interessiert, sondern konzentriert sich darauf, die Effizienz der bestehenden Prozesse zu verbessern. Der Analyzer folgt dem Prospector zwar in neue Märkte, strebt aber wie der Defender nach Sicherheit, in dem das Risiko für Produkte und Märkte durch verstärkte Überwachung minimiert wird. Analyzer tendieren dazu, eine Second Mover-Strategie zu bevorzugen. Als vierter Typ ist sich der Reactor zwar über die Unsicherheit der Umwelt bewusst, hat aber keine stabile Strategie, da er entweder dem Markt schnell folgt oder eine abwartende Haltung einnimmt.

In Bezug auf die Medienbranche entwickelt Chan-Olmsted (2006) eine Typologie der Adoption von Medientechnologien, die auf der Miles-Snow-Typologie basiert. Innovative Prospectors experimentieren innovativ und proaktiv mit neuen Produkten und suchen nach Chancen in neuen Märkten. Unternehmerische bzw. Entrepreneurial Prospectors investieren aggressiv und risikofreudig in neue Produkte und/oder Märkte, während unternehmerische bzw. Entrepreneurial Analyzers innerhalb eines stabilen Rahmens ihrer gegebenen Struktur und Prozesse vorsichtig nach neuen Chancen durch neue Technologien suchen. Conservative Analyzers warten lange ab und übernehmen neue Technologien nur langsam. Defender warten so lange ab, bis sich eine neue Technologie auf dem Markt etabliert hat, um sie zu übernehmen. Und zuletzt die Reactors, die entweder versuchen, von den neuen Technologien in einem sehr frühen Stadium zu profitieren oder abwarten, bis die Technologie in ihrer Branche weit verbreitet und etabliert ist.

Rogers (2003) unterscheidet in seinem Text zur Diffusionsforschung „Diffusion of innovation“ zwischen Innovators, Early Adopters, Early Majority, Late Majority und Laggards. Innovators sind technologiebegeistert, haben hohe finanzielle Ressourcen und sind risikofreudig, wodurch sie Innovationen als Erstes adaptieren. Early Adopters gelten als Visionäre und werden von Projekten mit hohem Risiko und hoher 
Belohnung angezogen. Sie investieren in Innovationen und üben Einfluss auf die nachfolgenden Gruppen. Die Early Majority ist nicht so innovationsfreudig wie die Early Adopters, lässt sich aber schnell von den Vorteilen technologischer Innovationen überzeugen. Sie können als Pragmatiker verstanden werden und warten so lange ab, bis sich eine neue Technologie auf dem Markt etabliert hat, um sie zu übernehmen. Die Late Majority ist konservativ und adaptiert erst dann neue Technologien, wenn der Zugehörigkeitsdruck so groß geworden ist, dass sie reagieren muss, um nicht abgehängt zu werden. Laggards sind Nachzügler und versuchen den Status Quo so lange wie möglich aufrecht zu halten. Sie adaptieren neue Technologien erst dann, wenn diese (von der Branche) bereits als überholt eingestuft werden.

Alle drei genannten Konstrukte eignen sich für eine Unterteilung der Medienunternehmen auf Basis ihrer Schnelligkeit und ihres Zeitpunkts der Technologieadoption. Während bei Miles und Snow (1978) und Chan-Olmsted (2006) der ausschlaggebende Faktor der Zuordnung zu einem Unternehmenstyp der Investitionszeitpunkt im Vergleich zur Konkurrenz und in Bezug auf das Auftauchen der Technologie ist, ist der ausschlaggebende Faktor bei Rogers (2003) der tatsächlichen Adoptionszeitpunkt einer Technologie in Bezug auf alle Investitionen und Akquisitionen in diese Technologie.

\subsection{Planungs- und Erwartungshorizont der Technologieadoption}

Der Planungshorizont wird in der strategischen Planung als Grad der Zukunftsorientierung verstanden und gibt an, wie weit in die Zukunft Unternehmen planen (Goodman 1973). Üblicherweise werden drei Planungshorizonte unterschieden: kurz-, mittel- und langfristig (Schumann et al. 2014). Der kurzfristige Planungshorizont bezieht sich in der Regel auf einen Planungshorizont von bis zu einem Jahr. Die mittelfristige Planung geht meist von einem Planungshorizont zwischen einem und fünf Jahren aus und der langfristige Planungshorizont kann oft einen Zeitraum von fünf bis zehn oder sogar über 20 Jahre umfassen (Schumann et al. 2014; SołoduchoPelc 2015; Hubert 2016). Häufig wird zwischen strategischer (langfristiger), taktischer (mittelfristiger) und operativer (kurzfristiger) Planung unterschieden (Hubert 2016).

Die Verfügbarkeit von strategierelevanten Informationen über die Zukunft ist ein wichtiger Faktor für die strategische Planung. Je weiter ein Unternehmen in die Zukunft blickt und plant, desto größer ist die mit dem Plan verbundene Unsicherheit (Schumann et al. 2014; Wilczek 2019). Risiko und Unsicherheit beeinflussen - wie auch den Adoptionszeitpunkt - den Planungshorizont maßgeblich. Reichhaltiges Wissen über die Umwelt der Organisation ist daher ein relevanter Faktor für organisatorische Zeithorizonte und Planungsstrategien, um der Unsicherheit zu begegnen. Ein reichhaltiges und differenziertes Wissen über die Umwelt ermöglicht es Unternehmen, sich an neue Gegebenheiten im Markt sinnvoll anzupassen. Goodman (1973) liefert eine Typologie von vier Organisationstypen, die auf ihrem Wissen über die Umwelt und ihren Zeithorizont basiert. Naive Unternehmen haben weder Wissen über ihr gegenwärtiges Umfeld noch orientieren sie sich an einer langfristigen Planung. Der Erfolg dieser Unternehmen ist zufällig und basiert auf einem ,Trial and Error"-Prinzip. Idealistic Unternehmen fokussieren und orientieren sich in ihrem 
strategischen Verhalten an der Zukunft, obwohl sie wenig Wissen über ihre Umwelt haben. Die Annahme einer stabilen Gegenwart ist sowohl eine Grundvoraussetzung als auch eine große Gefahr für diesen Organisationstypen. Professional-technical Organisationen verfügen über reichhaltiges Wissen über ihre aktuelle Umwelt, neigen aber dazu, sich bei ihrer strategischen Planung auf kurzfristige Zeithorizonte zu konzentrieren. Dieser Organisationstyp passt sich nur langsam an Innovationen an. Professional-sophisticated Organisationen haben ein reichhaltiges Wissen über ihr gegenwärtiges Umfeld und richten ihr strategisches Verhalten auf zukünftige Aspekte aus. Diese Unternehmen gehen von verschiedenen Zukunftsszenarien aus und passen ihre Planung diesen an. Hierdurch sind sie auf verschiedene Veränderungen der zukünftigen Gegebenheiten vorbereitet (Goodman 1973).

Darüber hinaus beeinflussen die Unternehmensstruktur, -größe und das -alter die strategische Planung. Unterschiedliche Eigentumsstrukturen setzen spezifische Anreize und Einschränkungen und prägen damit die Entscheidungsfindung der Akteure auf besondere Weise (Letts et al. 1997; Slater und Mohr 2006; Zellweger 2007; Lumpkin und Brigham 2011; Reilly et al. 2016; Wilczek 2019). So haben beispielsweise Familienunternehmen und Non-Profit-Organisationen einen längerfristigen Zeithorizont als börsennotierte Unternehmen (Letts et al. 1997; Zellweger 2007). Zudem hat die Größe eines Unternehmens einen wesentlichen Einfluss auf den Planungshorizont (Smith 1998; Stonehouse und Pemberton 2002; Kraus et al. 2006; Deimel 2008; Reilly et al. 2016): Während kleine und mittlere Unternehmen einen kurz- oder mittelfristigen Planungshorizont vorweisen (Stonehouse und Pemberton 2002; Deimel 2008), weil sie flexibler auf Umweltentwicklungen reagieren müssen (Smith 1998; Kraus et al. 2006), haben größere Unternehmen einen langfristigen Planungshorizont (Reilly et al. 2016). Betrachtet man den strategischen Planungshorizont von Startups im Vergleich zu älteren, etablierten Unternehmen, gibt es einige Studien, die sich auf das Alter von Unternehmen und ihr Innovationpotenzial konzentrieren (Hansen 1992; Balasubramanian und Lee 2008; Loderer und Waelchli 2010). So argumentieren Balasubramanian und Lee (2008), dass ein höheres Unternehmensalter negativ mit der Innovationsfähigkeit zusammenhängt.

Unternehmen, deren Handeln auf einem kurzfristigen Planungshorizont basiert, versäumen Zeit in neue Entwicklungen von und in die Übernahme neuer Technologien $\mathrm{zu}$ investieren, um sich Wettbewerbsvorteile zu verschaffen (Mezger und Violani 2011; Sołoducho-Pelc 2015). Der Fokus auf einen langfristigen Planungshorizont erleichtert die ständige Suche nach neuen Entwicklungsperspektiven, garantiert das Gleichgewicht zwischen aktuellen Ressourcen und dem Zukunftspotenzial und minimiert dadurch Unsicherheit (Sołoducho-Pelc 2015).

\section{Vorgehensweise und Methodik}

Die hier durchgeführte Analyse beruht auf den Daten der Plattform Crunchbase (Crunchbase 2021a), welche von einer Reihe von Venture-Partnern monatlich eingereicht und überwacht werden. Zudem werden die Crunchbase-Daten von über 400 internen Algorithmen durchsucht und validiert (Crunchbase 2021b). Der Datensatz ermöglicht es, Technologieinvestitionen und -akquisitionen - im vorliegenden 
Fall von Medienkonzernen - zu untersuchen. Die Auswahl der zu betrachtenden Technologien wurde anhand des Gartner Hype Cycle of Emerging Technologies (GHC) vorgenommen. Dabei wurden alle Technologien berücksichtigt, die seit dem Jahr 2000 auf dem GHC gelistet wurden. Die vorliegende Datenbasis umfasst 3002 Technologieinvestitionen (2550 Beteiligungsinvestitionen und 452 Akquisitionen) von Medienkonzernen in 39 Emerging Technologies aus dem Jahren 2000 bis 2019.

Als Medienunternehmen werden in der Untersuchung Unternehmen verstanden, deren Kerngeschäft in der Produktion und/oder dem Vertrieb von Medieninhalten liegt (Voci et al. 2019). Darunter fallen auch Unternehmen, die nicht nur reine Technikprovider sind, sondern große Teile ihres Umsatzes mit Erlösen aus Rechten, Lizenzen und/oder Werbung erwirtschaften und Einfluss auf die „kommunikative Umwelt" haben (Institut für Medien- und Kommunikationspolitik 2020). Für die Analyse wurden 100 Medienunternehmen aus einem jährlich erscheinenden Ranking der (nach Umsatz) 100 größten Medienunternehmen der Welt im Jahr 2018 des Instituts für Medien- und Kommunikationspolitik (2020; Simon 2019) ausgewählt.

Der GHC wird im Rahmen dieser Untersuchung nicht nur zur Auswahl und Definition der Technologien herangezogen. Zudem wurde der GHC für die Analyse der Daten verwendet. Im GHC durchlaufen die Technologien fünf Phasen (Fenn und Raskino 2008), vom Aufkommen einer Technologie bis zur Produktivität (Innovation Trigger, Peak of Inflated Expectations, Trough of Disillusionment, Slope of Enlightenment und Plateau of Productivity). Zudem wird im GHC jeder Technologie ein Reifehorizont zugeordnet, da sich die technologischen Innovationen mit unterschiedlicher Geschwindigkeit durch den Hype Cycle bewegen (Fenn et al. 2013). Dieser Reifehorizont bezieht sich auf die Zeitspanne, die eine Technologie benötigt, um von ihrer aktuellen Position im Hype Cycle aus das Plateau of Productivity zu erreichen und zu einem Standard auf dem Markt zu werden (less than 2 years, 2 to 5 years, 5 to 10 years, more than 10 years und obsolete before plateau).

Um in der vorliegenden Untersuchung die Frage von Adoptionszeitpunkt und -schnelligkeit zu beantworten, wurde zum einen die Zeitspanne zwischen dem erstmaligen Erscheinen der VR- oder AR-Technologie auf dem GHC und dem Investitionszeitpunkt (aus den Crunchbase-Daten) ermittelt. Somit sind Aussagen dazu möglich, wie früh oder spät Medienunternehmen in die (neuen) Technologien AR und VR investieren. Neben dieser Zeitspanne wird identifiziert, in welcher Phase sich AR und VR auf dem GHC befanden, als die Medienunternehmen in diese Technologien investiert haben. Hieraus lässt sich eine qualitative Komponente der Frühzeitigkeit ermitteln. Sie gibt Aufschluss darüber, welche Relevanz die Technologie zum Investitionszeitpunkt hatte bzw. welche Erwartungen an diese zum Investitionszeitpunkt gestellt wird.

Der Planungshorizont der Unternehmen bei der Technologieinvestition wird durch den Reifehorizont der Technologie auf dem GHC zum Zeitpunkt der Investition eines Medienunternehmens in ein AR-/VR-Startup/Unternehmen ermittelt. Es ist somit möglich, Aussagen darüber zu treffen, wie weit und mit wie viel „Unsicherheit“ die Unternehmen in die Zukunft bei der jeweiligen Technologieinvestition geplant haben bzw. welchen Erwartungshorizont hinsichtlich des Erreichens eines Produktivitätslevels ein Unternehmen an eine Technologieinvestition anlegt. 


\section{Empirische Ergebnisse zu Investitionen in Augmented und Virtual Reality}

Von den 3002 durch Medienunternehmen getätigten Technologieinvestitionen (2550 Beteiligungsinvestitionen, 452 Akquisitionen) beziehen sich 178 auf AR oder VR (inkl. Virtual World). Diese teilen sich auf in 72 auf AR (64 Beteiligungsinvestitionen, 8 Akquisitionen) und 106 auf VR (94 Beteiligungsinvestitionen, 12 Akquisitionen). Das sind in Summe ca. $6 \%$ der betrachteten Technologieinvestitionen (Tab. 1).

Tab. 1 Beschreibung der Stichprobe, Technologieinvestition in Emerging Technologies, AR, VR

\begin{tabular}{llll}
\hline & Beteiligungsinvestitionen & Akquisitionen & Gesamt \\
\hline Emerging Technologies $^{a}$ & 2550 & 452 & $3002(100 \%)$ \\
... davon in AR & 64 & 8 & 72 \\
... davon in VR & 94 & 12 & 106 \\
Summe AR und VR & $158(6 \%)$ & $20(4 \%)$ & $178(6 \%)$ \\
\hline
\end{tabular}

${ }^{\mathrm{a} E m e r g i n g ~ T e c h n o l o g i e s: ~ 3 D ~ P r i n t i n g, ~ A r t i f i c i a l ~ I n t e l l i g e n c e, ~ A u g m e n t e d ~ R e a l i t y, ~ B i g ~ D a t a, ~ B i o m e t r i c s, ~}$ Biotechnology, Bitcoin, Cloud Computing, Cryptocurrency, Drones, Gamification, Image Recognition, Internet of Things, Linux, Location Based Services, Machine Learning, mHealth, Mobile Payments, Natural Language Processing, NFC, Predictive Analytics, Private Cloud, Prediction Markets, QR Codes, Quantified Self, RFID, SaaS, Semantic Web, Speech Recognition, Text Analytics, Unified Communications, Video Conferencing, Virtual Assistant, Virtual Currency, Virtual Reality, Virtual World, VoIP, Wearables, Wireless

Tab. 2 AR-und VR-Technologieinvestitionen im Zeitverlauf (2000 bis 2019)

\begin{tabular}{llll}
\hline & AR & VR & Gesamt \\
\hline 2000 & 0 & 0 & 0 \\
2001 & 0 & 0 & 0 \\
2002 & 0 & 0 & 0 \\
2003 & 0 & 0 & 0 \\
2004 & 1 & 1 & 2 \\
2005 & 0 & 1 & 1 \\
2006 & 0 & 0 & 0 \\
2007 & 1 & 1 & 2 \\
2008 & 0 & 0 & 0 \\
2009 & 0 & 2 & 2 \\
2010 & 0 & 2 & 2 \\
2011 & 0 & 0 & 0 \\
2012 & 2 & 0 & 2 \\
2013 & 7 & 5 & 12 \\
2014 & 6 & 7 & 13 \\
2015 & 8 & 17 & 25 \\
2016 & 16 & 21 & 37 \\
2017 & 16 & 36 & 52 \\
2018 & 12 & 10 & 22 \\
2019 & 3 & 3 & 6 \\
Summe & $\mathbf{7 2}$ & $\mathbf{1 0 6}$ & $\mathbf{1 7 8}$ \\
\hline & & &
\end{tabular}


Tab. 3 Verteilung der AR- und VR-Technologieinvestitionen auf die untersuchten Medienunternehmen

\begin{tabular}{|c|c|c|c|c|c|}
\hline & $\begin{array}{l}\text { Häufigkeit } \\
\text { AR }\end{array}$ & $\begin{array}{l}\text { Häufigkeit } \\
\text { VR }\end{array}$ & $\begin{array}{l}\text { Häufigkeit } \\
\text { Gesamt }\end{array}$ & Prozent & $\begin{array}{l}\text { Kumulierte } \\
\text { Prozent }\end{array}$ \\
\hline Alphabet Inc & 6 & 15 & 21 & 12 & 12 \\
\hline Microsoft Corporation & 12 & 6 & 18 & 10 & 22 \\
\hline Comcast & 4 & 11 & 15 & 8 & 30 \\
\hline $\begin{array}{l}\text { Bertelsmann SE \& Co. } \\
K G a A\end{array}$ & 3 & 9 & 12 & 7 & 37 \\
\hline Alibaba Group & 8 & 3 & 11 & 6 & 43 \\
\hline $\begin{array}{l}\text { News Corp./21st Century } \\
\text { Fox }\end{array}$ & 4 & 7 & 11 & 6 & 49 \\
\hline$A T \& T$ & 2 & 8 & 10 & 6 & 55 \\
\hline Discovery Inc & 5 & 4 & 9 & 5 & 60 \\
\hline NetEase & 3 & 3 & 6 & 3 & 63 \\
\hline Baidu Inc & 1 & 4 & 5 & 3 & 66 \\
\hline Oath/Verizon Media & 3 & 2 & 5 & 3 & 69 \\
\hline Tencent Holdings Ltd & 2 & 3 & 5 & 3 & 72 \\
\hline The Hearst Corporation & 1 & 4 & 5 & 3 & 75 \\
\hline Facebook & 2 & 2 & 4 & 2 & 77 \\
\hline Bonnier $A B$ & 2 & 2 & 4 & 2 & 79 \\
\hline Apple & 2 & 1 & 3 & 2 & 81 \\
\hline $\begin{array}{l}\text { MGM Holdings/MGM } \\
\text { Studios }\end{array}$ & 0 & 3 & 3 & 2 & 83 \\
\hline Modern Times Group & 0 & 3 & 3 & 2 & 84 \\
\hline Sony Entertainment & 1 & 2 & 3 & 2 & 86 \\
\hline The Walt Disney Company & 2 & 1 & 3 & 2 & 88 \\
\hline $\begin{array}{l}\text { Nielsen Holdings plc/ } \\
\text { Nielsen }\end{array}$ & 1 & 1 & 2 & 1 & 89 \\
\hline Axel Springer SE & 1 & 1 & 2 & 1 & 90 \\
\hline Cox Enterprises Inc & 1 & 1 & 2 & 1 & 91 \\
\hline Liberty, Qurate Retail, Inc & 1 & 1 & 2 & 1 & 92 \\
\hline $\begin{array}{l}\text { National Amusements: } \\
\text { Viacom, CBS }\end{array}$ & 0 & 2 & 2 & 1 & 93 \\
\hline Nintendo Company Ltd & 1 & 1 & 2 & 1 & 94 \\
\hline $\begin{array}{l}\text { Sega/Sega Sammy Hol- } \\
\text { dings }\end{array}$ & 1 & 1 & 2 & 1 & 96 \\
\hline Shanghai Media Group & 0 & 2 & 2 & 1 & 97 \\
\hline $\begin{array}{l}\text { Tokyo Broadcasting System } \\
\text { Holdings, Inc }\end{array}$ & 1 & 1 & 2 & 1 & 98 \\
\hline AMC Networks & 0 & 1 & 1 & 1 & 98 \\
\hline Asahi Shimbun Company & 1 & 0 & 1 & 1 & 99 \\
\hline Grupo Globo & 1 & 0 & 1 & 1 & 99 \\
\hline ProSiebenSat.1 SE & 0 & 1 & 1 & 1 & 100 \\
\hline Amazon & 0 & 0 & 0 & - & 100 \\
\hline Summe & 72 & 106 & 178 & 100 & - \\
\hline
\end{tabular}


Eine substanzielle Anzahl an Investitionen ist dabei ab $2013 \mathrm{zu}$ beobachten (Tab. 2), wobei ab 2015 insbesondere die Anzahl an Investitionen in VR-Startups stark steigt und 2017 mit 52 Investitionen (11\% aller analysierten Technologieinvestitionen in diesem Jahr) ihren Höhepunkt erreicht. 2018 geht die Zahl der Investitionen in AR und VR wieder deutlich zurück.

Ein Blick auf die investierenden Unternehmen zeigt, dass zwei Drittel (66\%, 118) der 178 Investitionen in AR oder VR von nur zehn Unternehmen durchgeführt wurden. Knapp ein Drittel $(30 \%, 54)$ der 178 Investitionen stammt von nur drei Investoren. Es ist demnach eine starke Konzentration von Technologieinvestitionen auf wenige (große) Medienunternehmen zu beobachten (Tab. 3). Die TopInvestoren (gemessen an der Anzahl der Technologieinvestments) in AR- und VRStartups sind Alphabet und Microsoft, wobei Alphabet stärker in VR, Microsoft stärker in AR investiert. Microsoft und Alibaba sind bei den Top-Investoren die einzigen Unternehmen, die öfter in AR investieren als in VR. Neben den bereits erwähnten Unternehmen sind Comcast, Bertelsmann, News Corporation/21 Century Fox, AT\&T und Discovery die Top-Investoren. Es sind somit nicht nur die Big-5 GAFAM-Konzerne (Facebook ist nur mit 4 Investitionen, Apple nur mit 3, Amazon mit 0 Investitionen vertreten), sondern auch Unternehmen, die eher (auch traditionell) dem Bereich der Inhaltserstellung zuzurechnen sind.

\subsection{Schnelligkeit und Zeitpunkt der Technologieinvestition}

Von den 178 Investitionen in AR und VR entfallen mehr als 50\% (97) auf die dritte Phase (Trough of Disillusionment) des GHC, weitere ca. 30\% (55) auf die vierte Phase (Slope of Enlightenment) des GHC (Tab. 4). Die untersuchten Unternehmen investieren somit hauptsächlich nach dem Peak of Inflated Expectations in AR und VR, also dann, wenn die ersten (ursprünglichen) Erwartungen an AR- und VRTechnologien nicht erfüllt werden konnten und die Berichterstattung über AR und VR massiv abgenommen hat. Dennoch wird in der Slope of Enlightenment-Phase weiter an der Entwicklung von AR- und VR-Anwendungen gearbeitet, wodurch sich langsam praktische und funktionierende Technologieanwendungen abzeichnen.

Sucht man nach Unterschieden zwischen den konkreten investierenden Unternehmen sowie deren Herkunftsländern, fällt auf, dass US-amerikanische, chinesische

Tab. 4 Verteilung der AR- und VR-Technologieinvestitionen auf die Phasen des GHC

\begin{tabular}{llll}
\hline & Häufigkeit & Prozent & Kumulierte Prozente \\
\hline $\begin{array}{l}\text { Vor erstmaligem Erscheinen der Technologie } \\
\text { auf GHC }\end{array}$ & 7 & 4 & 4 \\
Innovation Trigger & 2 & 1 & 5 \\
Peak of Inflated Expectations & 2 & 1 & 6 \\
Trough of Disillusionment & 97 & 54 & 61 \\
Slope of Enlightenment & 55 & 31 & 92 \\
Plateau of Productivity & 13 & 7 & 99 \\
Nachdem Technologie nicht mehr auf GHC & 2 & 1 & $\mathbf{1 0 0}$ \\
vertreten & & & \\
Summe & $\mathbf{1 7 8}$ & $\mathbf{1 0 0}$ & $\mathbf{-}$ \\
\hline
\end{tabular}


Tab. 5 Schnelligkeit (Frühzeitigkeit) der AR- und VR-Technologieinvestitionen

\begin{tabular}{llllll}
\hline $\begin{array}{l}\text { Differenz von Auftauchen der } \\
\text { Technologie auf dem GHC und } \\
\text { Investitionszeitpunkt }\end{array}$ & $\begin{array}{l}\text { Häufigkeit } \\
\text { AR }\end{array}$ & $\begin{array}{l}\text { Häufigkeit } \\
\text { VR }\end{array}$ & $\begin{array}{l}\text { Häufigkeit } \\
\text { Gesamt }\end{array}$ & $\begin{array}{l}\text { Prozent } \\
\text {-9 Jahre }\end{array}$ & $\begin{array}{l}\text { Kumulierte } \\
\text { Prozente }\end{array}$ \\
-6 Jahre & 0 & 1 & 1 & 1 & 1 \\
-4 Jahre & 0 & 1 & 1 & 1 & 1 \\
-3 Jahre & 0 & 1 & 1 & 1 & 2 \\
-2 Jahre & 0 & 1 & 2 & 1 & 3 \\
0 Jahre & 1 & 5 & 1 & 1 & 3 \\
1 Jahre & 0 & 7 & 7 & 3 & 7 \\
2 Jahre & 0 & 18 & 18 & 4 & 11 \\
3 Jahre & 1 & 20 & 21 & 10 & 21 \\
4 Jahre & 0 & 35 & 35 & 20 & 32 \\
5 Jahre & 0 & 10 & 10 & 6 & 58 \\
6 Jahre & 0 & 3 & 3 & 2 & 60 \\
8 Jahre & 2 & 0 & 2 & 1 & 61 \\
9 Jahre & 7 & 1 & 8 & 4 & 65 \\
10 Jahre & 6 & 1 & 7 & 4 & 69 \\
11 Jahre & 8 & 0 & 8 & 4 & 74 \\
12 Jahre & 16 & 0 & 16 & 9 & 83 \\
13 Jahre & 16 & 0 & 16 & 9 & 92 \\
14 Jahre & 12 & 0 & 12 & 7 & 98 \\
15 Jahre & 3 & 0 & 3 & 2 & $\mathbf{1 0 0}$ \\
Summe & $\mathbf{7 2}$ & $\mathbf{1 0 6}$ & $\mathbf{1 7 8}$ & $\mathbf{1 0 0}$ & - \\
\hline & & & & &
\end{tabular}

und deutsche Konzerne (für alle weiteren Länder ist für Vergleiche die Fallzahl zu klein) hauptsächlich in der dritten und vierten GHC-Phase investieren.

Betrachtet man die Frühzeitigkeit und Schnelligkeit der Reaktion anhand der Differenz zwischen dem Auftauchen der Technologie auf dem GHC und dem Investitionszeitpunkt (Tab. 5), fällt auf, dass es einige sehr wenige Unternehmen gibt, die unmittelbar nach Auftreten, teilweise bereits vor Auftreten der Technologien auf dem GHC investieren. Rund $40 \%$ der Investitionen entfallen jedoch in den Zeitraum von 2 bis zu 4 Jahren nach Auftauchen der Technologien, ca. 25\% der Investitionen erfolgen erst nach 12 bis 14 Jahren. Hierdurch lassen sich zwei Investitionsphasen identifizieren. Auffällig ist dabei, dass - bezogen auf die bekannten Kategorien nach Rogers (2003) - diese beiden Innovationsphasen der Early Majority und Late Majority zuordenbar sind. Die erste Gruppe ist zwar nicht so innovationsfreudig wie die Innovators oder Early Adopters, lässt sich aber (als große Gruppe) schnell von technologischen Innovationen und deren Vorteilen überzeugen und investiert zeitgleich mit vielen anderen auch in die Technologien. Die spätere Gruppe lässt sich der Late Majority zuordnen. Diese Unternehmen sind eher konservativ und warten auf neue Versionen oder Verbesserungen der Technologien und Anwendungen, bis sie in AR und VR investieren.

Differenziert man nach den konkret investierenden Unternehmen sowie deren Herkunftsländern lassen sich deutsche Konzerne - im Gegensatz zu den US-amerika- 
nischen und chinesischen (wobei diese Beobachtung aufgrund der geringen Fallzahl deutscher Medienkonzerne mit Vorsicht interpretiert werden sollte) - am wenigsten der Late Majority zuordnen. Deutsche Konzerne investieren demnach - wenn sie investieren (und dies tun sie deutlich seltener als die US-amerikanischen und chinesischen Konzerne) - eher als Early Majority. Kleinere vereinzelte Unterschiede zeigen sich bei den einzelnen Unternehmen: Während die Investitionen von Comcast breit über die Gruppen und Typen streuen, spiegeln die Investitionen von Alphabet die Gruppe der Innovators am ehesten wider. Alibaba hingegen ist mit seinen Technologieinvestitionen zurückhaltender und neigt dazu, den Eigenschaften der Late Majority zu entsprechen. Weitere Auffälligkeiten sind hier jedoch nicht zu identifizieren, was auch auf die Verteilung der GHC-Phasen zutrifft.

Sehr auffällig sind jedoch Unterschiede, wenn man zwischen den beiden untersuchten Technologien differenziert (Tab. 5): 58 von 72 AR-Investitionen erfolgen 10 bis 14 Jahre nach erstmaligem Auftauchen der Technologie auf dem GHC. Die 106 VR-Investitionen verteilen sich hauptsächlich auf 2 bis 5 Jahre (83 Fälle) nach erstmaligen Erscheinen der Technologie auf dem GHC. Im Fall von VR investieren die Unternehmen demnach deutlich früher (bezogen auf den Erscheinungszeitpunkt einer neuen Technologie), im Fall von AR warten die Unternehmen sehr lange, bis eine Investition erfolgt.

\subsection{Planungs- und Erwartungshorizont der Technologieinvestition}

Von den 178 AR- und VR-Investitionen kann bei 168 ein Planungshorizont erhoben werden (Tab. 6). Auffällig ist, dass keine Investition zu einem Zeitpunkt erfolgt, zu dem das Erreichen der Produktivitätslevels in weniger als zwei Jahren erwartet wurde. Aber auch nur in einem einzigen Fall wurde eine Investition getätigt, die auf einen Planungshorizont (Erreichen des Produktivitätslevels) von mehr als 10 Jahren ausgerichtet war. Vielmehr bewegen sich alle Investitionen in einem mittleren Planungshorizont, wobei sich über drei Viertel der Investitionen auf einen Planungshorizont von 5 bis 10 Jahren aufteilen, alle weiteren Investitionen (knapp ein Viertel) auf einen Zeitraum von 2 bis 5 Jahren. Die analysierten Unternehmen konnten zum Zeitpunkt ihrer Investition weder davon ausgehen, dass sich innerhalb kurzer Zeit bereits Produktivitätseffekte einstellen, noch wurde sehr weit (mehr als zehn Jahre) in die Zukunft investiert.

Tab. 6 Verteilung der AR- und VR-Technologieinvestitionen auf die Planungshorizonte bzw. Reifehorizonte im GHC

\begin{tabular}{|c|c|c|c|c|c|c|}
\hline & & $\begin{array}{l}\text { Häufigkeit } \\
\text { AR }\end{array}$ & $\begin{array}{l}\text { Häufigkeit } \\
\text { VR }\end{array}$ & $\begin{array}{l}\text { Häufigkeit } \\
\text { Gesamt }\end{array}$ & Prozent & $\begin{array}{l}\text { Kumulierte } \\
\text { Prozente }\end{array}$ \\
\hline $\begin{array}{l}\text { Kurzfristiger } \\
\text { Planungshorizont }\end{array}$ & $\begin{array}{l}\text { Less than } \\
2 \text { years }\end{array}$ & 0 & 0 & 0 & 0 & 0 \\
\hline \multirow{2}{*}{$\begin{array}{l}\text { Mittelfristiger } \\
\text { Planungshorizont }\end{array}$} & 2 to 5 years & 0 & 35 & 35 & 21 & 21 \\
\hline & $\begin{array}{l}5 \text { to } \\
10 \text { years }\end{array}$ & 70 & 62 & 132 & 79 & 99 \\
\hline \multirow[t]{2}{*}{$\begin{array}{l}\text { Langfristiger } \\
\text { Planungshorizont }\end{array}$} & $\begin{array}{l}\text { More than } \\
10 \text { years }\end{array}$ & 1 & 0 & 1 & 1 & 100 \\
\hline & Summe & 71 & 97 & 168 & 100 & - \\
\hline
\end{tabular}


Ein interessantes Ergebnis zeigt sich, wenn man die einzelnen Jahre der Investitionen näher betrachtet. Von den 37 Investitionen aus dem Jahr 2017 sind alle (35, für zwei Investitionen konnte kein Planungshorizont zugeordnet werden) einem Planungshorizont von 2 bis 5 Jahren zuzuordnen. In allen anderen Jahren, in welchen in die beiden Technologien investiert wurde, sind bis auf eine einzige Ausnahme immer Planungshorizonte von 5 bis 10 Jahren zu identifizieren. Möglicherweise war 2017 ein Jahr, in dem den Technologien besonders viel ,zugetraut“" wurde (ein kürzerer Zeitraum bis zum Erreichen des Produktivitätslevels). Schließlich war 2017 auch das Jahr, in dem mit Abstand die meisten Investitionen in diese Technologien erfolgten. Differenziert man AR und VR, fällt auf, dass 70 von 71 Investitionen ein Planungshorizont von 5 bis 10 Jahren zuzuordnen ist (Tab. 6). Für VR entfallen auf diesen Planungshorizont zwei Drittel der Investitionen, ein Drittel entfällt auf den Planungshorizont von 2 bis 5 Jahren. AR-Investitionen werden offenbar zu Zeitpunkten getätigt, in denen der Erwartungshorizont bis das Produktivitätslevel erreicht wird, weiter entfernt ist, als bei zumindest vielen VR-Investitionen. Untersucht man Unterschiede einzelner Länder (Stammland des investierenden Konzerns) - man hätte ein unterschiedlich hohes Risikoverhalten vermuten können - wird zunächst noch einmal deutlich, dass der Großteil der Investitionen von US-amerikanischen Konzernen getätigt wird. Aufgrund der teilweise sehr kleinen Gruppengrößen sind Gruppenvergleiche zwischen den einzelnen Ländern nicht sinnvoll bzw. möglich. Die weitere differenzierte Betrachtung der Unternehmen zeigt keine Auffälligkeiten: Zum einen sind die Gruppengrößen zu klein, um sinnvoll Gruppenvergleiche vornehmen zu können. Zum anderen sind bei den Top-Investoren (mit einer substanziellen Anzahl an Investitionen in die beiden Technologien) keine Unterschiede erkennbar: Es geht um Planungshorizonte von 2 bis 10 Jahren, wobei immer häufiger auf 5 bis 10 Jahre (als auf 2 bis 5 Jahre) verwiesen werden kann.

\section{Diskussion der Ergebnisse}

Einen grundsätzlichen Adoptionszeitpunkt von AR- und VR-Anwendungen und -Technologien scheint es nicht zu geben. Besonders die Unsicherheit der Rentabilität sowie das strategische Verhalten von Konkurrenten beeinflussen den Investitionszeitpunkt sowie den Planungshorizont von Medienunternehmen (Reinganum 1981; Jensen 1982; Balcer und Lippman 1984; Bhattacharya et al. 1986). Aufgrund breit gefächerter Anwendungsgebiete, Branchen, die fundamental verändert werden können, sowie noch nicht vollständig ausgeschöpfter Nutzungspotenziale sind AR und VR als disruptive Technologien zu verstehen (Goldman Sachs Group 2016; Mütterlein et al. 2017; Engelmann 2018).

Obwohl ca. $6 \%$ aller untersuchter Technologieinvestitionen von Medienunternehmen auf AR- und VR-Anwendungen und -Technologien entfallen, zeigt sich dennoch ein differenziertes Bild über das strategische Verhalten von Medienunternehmen. Beide Technologien haben in der Medienbranche ein breites Anwendungsfeld gefunden, dennoch scheinen Medienunternehmen wenig investitionsfreudig zu sein. Es sind nur wenige Medienkonzerne, die in AR und VR investieren (im Sinne von Beteiligungen und Akquisitionen). Neben den bekannten Big-5 GAFAM-Konzernen 
(in diesem Fall besonders Google, Apple und Microsoft), sind unter den Investoren Medienkonzerne zu finden, die eher (traditionell) dem Bereich der Inhaltserstellung zuzurechnen sind.

Möchte man die Ergebnisse der Miles-Snow-Typologie (1978) zuordnen, können Medienunternehmen als Analyzer identifiziert werden. Im Gegensatz zu den Prospectors, die sehr früh und sehr vorausschauend in AR- und VR-Technologien investieren würden, treten die untersuchten Medienkonzerne mit der Investition in die Technologien zwar in neue Märkte ein, streben aber weiterhin nach Sicherheit, in dem sie erst in die Technologien investieren, wenn die hohen Erwartungen und die übermäßige Berichterstattung abgeflacht sind und eine Weiterentwicklung der Technologien absehbar ist. Analog dazu lassen sich die Medienunternehmen in der Typologie nach Chan-Olmsted (2006) den Entrepreneurial Analyzers zuordnen, die innerhalb eines stabilen Rahmens ihrer gegebenen Struktur und Prozesse vorsichtig nach neuen Chancen durch neue Technologien suchen.

Mithilfe der Typologie nach Rogers (2003) lassen sich die Medienunternehmen aufgrund ihres Investitionsverhaltens noch differenzierter betrachten. Die untersuchten Medienkonzerne investieren vor allem dann in VR und AR, wenn diese bereits auf dem GHC erschienen sind. Rund $40 \%$ der Investitionen entfallen in den Zeitraum von 2 bis zu 4 Jahren nach Auftauchen der Technologien; weitere über $25 \%$ der Investitionen erfolgen erst nach 12 bis 14 Jahren. Investitionen in einem Zeitraum von 2 bis 4 Jahren nach Auftauchen der Technologien auf dem GHC können als Early Majority-Investitionen bezeichnet werden. Medienunternehmen, die dieser Kategorie zugeordnet werden, sind zwar nicht so innovationsfreudig wie Innovators oder Early Adopters, lassen sich aber schnell von technologischen Innovationen und deren Vorteilen überzeugen. Die Medienunternehmen, die erst nach 12 bis 14 Jahren in AR oder VR investiert haben, können im Sinne der Typologie der Gruppe der Late Majority zugeordnet werden. Sie investieren erst in die Technologien, wenn bereits etablierte Anwendungen verfügbar sind und sie wettbewerbsfähig bleiben wollen. Auffällig ist dabei, dass Medienkonzerne, die in VR investieren, sich der Gruppe der Early Majority zuordnen lassen, während Medienunternehmen, die in AR investieren, das Verhalten der Late Majority aufweisen.

Die untersuchten Medienunternehmen planen bei den Investitionen in AR- und VR-Technologien und -Anwendungen vergleichsweise weit in die Zukunft. Drei Viertel der Investitionen wurden mit einem mittelfristigen Planungshorizont von 5 bis 10 Jahren getätigt. Dabei wurden AR-Investitionen offenbar zu Zeitpunkten getätigt, in denen der Erwartungshorizont bis zum Produktivitätslevel erreicht wird, weiter entfernt ist, als bei zumindest vielen VR-Investitionen. Somit planen Medienunternehmen bei AR weiter in die Zukunft als bei VR. So wird AR-Anwendungen ein höheres Marktpotenzial nachgesagt als VR-Anwendungen, da AR durch die einfachere Nutzungs- (ohne HMDs) sowie Integrationsmöglichkeiten zahlreiche Betätigungsfelder bietet (Kind et al. 2019).

Überträgt man die Ergebnisse in Goodmans Typologie (1973), so können die Medienkonzerne hinsichtlich ihrer Investitionen in AR- und VR-Anwendungen und -Technologien zwischen dem professional-technical und dem professional-sophisticated Organisationstyp eingeordnet werden. Diese Unternehmen haben ein reichhaltiges Wissen über ihr gegenwärtiges Umfeld, richten ihr strategisches Verhalten aber 
nicht ganz so weit auf zukünftige Aspekte aus wie der professional-sophisticated Organisationstyp. Sie sind somit nicht zwangsläufig auf verschiedene Zukunftsszenarien vorbereitet, wodurch Investitionen eher mittelfristig getätigt werden. Da die 100 größten Medienkonzerne (gemessen an ihrem Umsatz) betrachtet wurden, kann ein direkter Bezug zur Größe der Medienunternehmen hergestellt werden. So geht die Literatur davon aus, dass große (Medien-)Unternehmen einen langfristigen Planungshorizont verfolgen (Deimel 2008; Reilly et al. 2016). Die Ergebnisse bestätigen diese Annahme nur zum Teil: Die Daten zeigen, dass die 100 größten Medienunternehmen der Welt tendenziell einen mittelfristigen Planungshorizont ( 5 bis 10 Jahre) verfolgen, der bei AR sogar etwas länger ist als bei VR.

Die Ergebnisse liefern einige Handlungsimplikationen für Praktiker. Die hier untersuchten Medienunternehmen gehören zu den 100 größten und erfolgreichsten bzw. einflussreichsten Medienunternehmen der Welt (Simon 2019; Institut für Medien- und Kommunikationspolitik 2020). Ihre strategische Ausrichtung scheint damit erfolgreich zu sein. Andere (Medien-)Unternehmen können sich an deren Verhalten orientieren, wenn es um die Ausweitung der Kompetenzen hinsichtlich AR und VR geht. Die untersuchten Medienunternehmen reagieren zwar zögerlich auf die Technologien, sind aber dennoch aufgeschlossen, was sich in den Unternehmenstypen Analyzer und Entrepreneurial Analyzer widerspiegelt. Jedoch sind es aktuell nur sehr wenige Medienunternehmen in der Untersuchung, die in AR und VR investieren. Es scheint also (noch) kein „Big Thing“ in der Medienbranche zu sein oder es stecken nur sehr spezielle Nutzungsmotive dahinter. Den Ergebnissen zufolge, handelt es sich auch eher um technikaffinere Unternehmen wie Alphabet und Microsoft, die die meisten Investitionsbeteiligungen und Akquisitionen in AR und VR tätigen. Erst danach folgen klassische Medienunternehmen im weitesten Sinne wie Comcast und Bertelsmann. Aktuell sind beide Technologien ein Nischenthema in der Medienbranche, sofern es sich nicht um die Bereiche Video- und OnlineGames, Filme und zum Teil spezifische Marketing-Anwendungen handelt. Auffällig ist dabei, dass sich für VR relativ schnell geeignete Nutzungsmotive entwickelten, dies erklärt die Early Majority-Investitionen. Bei AR dagegen scheinen sich die Nutzungsmöglichkeiten für die Medienbranche sehr viel später nach erstmaligen Erscheinen herauszukristallisieren, wodurch Medienunternehmen Late Majority-Investitionen tätigen. Nichtsdestotrotz lohnt sich eine Beobachtung der Entwicklung von AR und VR in der Medienbranche, um - falls sich potenzielle Nutzungsmotive für ein Medienunternehmen herauskristallisieren - früh genug einsteigen zu können. Die hier untersuchten Medienunternehmen, die zwischen dem professional-technical und dem professional-sophisticated Organisationstyp nach Goodman (1973) liegen, informieren sich offenbar stark über ihr gegenwärtiges Umfeld, richten ihr strategisches Verhalten aber nicht ganz so weit auf zukünftige Aspekte. Der mittlere Planungshorizont, den die Medienunternehmen in der Untersuchung verfolgen, deutet darauf hin, dass die beiden Technologien ihr volles Potenzial noch nicht ausgeschöpft haben und weitere Nutzungsmöglichkeiten eröffnen. Der Erfolg von AR und VR in der Medienbranche ist abhängig von weiteren attraktiven Inhalten, die mithilfe der Technologien ausgespielt werden können. Aktuelle AR-/VR-Anwendungen zeichnen sich eher durch Pilotprojekte aus oder dienen Prestige-Zwecken, als dass sie in das Tagesgeschäft der Medienunternehmen einfließen. 
Einschränkungen der Ergebnisse dieser Arbeit liegen in folgenden Bereichen: Erstens basiert die Untersuchung zwar auf den weltweit 100 größten Medienunternehmen, doch besteht in dieser Stichprobe eine geringe Fallzahl an Technologieinvestitionen in AR und VR. Viele Berechnungen, wie beispielsweise Gruppenvergleiche zwischen den einzelnen Herkunftsländern der investierenden Medienunternehmen, sind aufgrund der Verteilung der Stichprobe bzw. der zu kleinen GröBe von Vergleichsgruppen kaum möglich. Zweitens ist die Crunchbase-Datenbank sehr „US-lastig“, wodurch Unternehmen aus anderen Ländern gegebenenfalls unterrepräsentiert sein könnten. Drittens können viele Startups/Unternehmen, in die investiert wurde, sowohl zu AR als auch zu VR zugeordnet werden bzw. bedienen technologisch beide Bereiche. Viertens ist unbekannt, was die Unternehmen mit den Technologien machen (oder machen werden): Die Ergebnisse müssen als eine Außenperspektive interpretiert werden. Es lassen sich keine Aussage darüber treffen, worauf die Investitionsentscheidungen beruhen. So kann nicht ausgeschlossen werden, dass (a) das strategische Verhalten eine Investitionsentscheidung im Hinblick auf eine spätere Beteiligungsrendite war, (b) Wettbewerber blockiert und/ oder Markteintrittsbarrieren aufgebaut werden sollten oder (c) die Investitionsentscheidung darauf abzielte, die Technologie im Unternehmen zu nutzen. Es ist zu bedenken, dass der Zeitpunkt der Technologieinvestition auch von Faktoren abhängig ist, die nicht (oder zumindest nur begrenzt) von den investierenden Unternehmen zu beeinflussen sind: So kann sich (obwohl die Entscheidung für eine Investition bereits länger zurückliegt und entsprechende Bemühungen bereits gestartet wurden) der Prozess beispielsweise durch Verhandlungen in die Länge ziehen und sich dadurch der Vollzug der Beteiligung oder Akquisition nach hinten verschieben. Ebenso beeinflussen externe Faktoren wie die Terminierung von Beteiligungs- bzw. Investitionsrunden den Zeitpunkt der Technologieinvestitionen. Zudem heißen die hier analysierten Investitionen nicht, dass sich die Unternehmen nicht auf andere Weise mit den Technologien beschäftigen und sich diese aneignen - beispielsweise durch internen Aktivitäten, wie Forschungs- und (Eigen-)Entwicklungsaktivitäten.

Im Zuge weiterer Untersuchungen zu den Technologieadoptionsstrategien von Medienunternehmen wäre interessant, die klein- und mittelständigen Medienunternehmen und deren strategisches Verhalten hinsichtlich neu aufkommender Technologien genauer zu betrachten. Denn ein Großteil der Medienunternehmen (dies trifft zumindest für die deutsche Medienlandschaft zu) gehören - im Gegensatz zu den hier betrachteten 100 größten Medienkonzernen der Welt - zu dieser Gruppe. Zudem kann, über die Analyse der Anzahl von Investitionen hinaus, auch eine Analyse der Investitionsvolumina in die Technologien und deren Zeitverlauf aufschlussreich sein. Hierdurch würden sich weitere Differenzierungen der Technologieadoptionsstrategien ausfindig machen lassen. Des Weiteren würde ein Blick in die Medienunternehmen Informationen darüber liefern, welche Zielsetzungen hinter den Investitionen stehen und was die Medienunternehmen mit den Technologien tatsächlich machen. Hieran anknüpfend stellt sich auch die Frage, wie die Technologien organisatorisch in die Medienunternehmen integriert werden. Zuletzt wäre besonders interessant, wie sich die Technologieinvestitionen und die daraus entstandenen Angebote - sowohl hinsichtlich der Akzeptanz und Durchsetzung am Markt als auch in finanzieller Hinsicht - auf den Erfolg der Medienunternehmen auswirken. 
Funding Open Access funding enabled and organized by Projekt DEAL.

Open Access Dieser Artikel wird unter der Creative Commons Namensnennung 4.0 International Lizenz veröffentlicht, welche die Nutzung, Vervielfältigung, Bearbeitung, Verbreitung und Wiedergabe in jeglichem Medium und Format erlaubt, sofern Sie den/die ursprünglichen Autor(en) und die Quelle ordnungsgemäß nennen, einen Link zur Creative Commons Lizenz beifügen und angeben, ob Änderungen vorgenommen wurden.

Die in diesem Artikel enthaltenen Bilder und sonstiges Drittmaterial unterliegen ebenfalls der genannten Creative Commons Lizenz, sofern sich aus der Abbildungslegende nichts anderes ergibt. Sofern das betreffende Material nicht unter der genannten Creative Commons Lizenz steht und die betreffende Handlung nicht nach gesetzlichen Vorschriften erlaubt ist, ist für die oben aufgeführten Weiterverwendungen des Materials die Einwilligung des jeweiligen Rechteinhabers einzuholen.

Weitere Details zur Lizenz entnehmen Sie bitte der Lizenzinformation auf http://creativecommons.org/ licenses/by/4.0/deed.de.

\section{Literatur}

Amit R, Zott C (2012) Creating value through business model innovation. MIT Sloan Management Review 53:41-49

Andal-Ancion A, Cartwright P, Yip G (2003) The digital transformation of traditional business. MIT Sloan Management Review 44:34-41

Azuma RT (1997) A survey of augmented reality. Presence: Teleoperators \& Virtual Environments 6:355-385

Balasubramanian N, Lee J (2008) Firm age and innovation. Ind Corp Change 17:1019-1047

Balcer Y, Lippman SA (1984) Technological expectations and adoption of improved technology. J Econ Theory 34:292-318

Berman SJ (2012) Digital transformation: opportunities to create new business models. Strategy and Leadership 40:16-24

Bhattacharya S, Chatterjee K, Samuelson L (1986) Sequential research and the adoption of innovations. Oxf Econ Pap 38:219-243

Bitkom e. V. (2019) Zukunft der Consumer Technology - 2019. Marktentwicklung, Trends, Mediennutzung, Technologien, Geschäftsmodelle. Bitkom e. V., Berlin

Casadesus-Masanell R, Zhu F (2013) Business model innovation and competitive imitation: The case of spon-sored-based business models. Strategic Management Journal 34:464-482

Chan-Olmsted SM (2006) Issues in media management and technology. In: Albarran AB, Chan-Olmsted SM, Wirth MO (Hrsg) Handbook of media management and economics. Lawrence Erlbaum, Mahwah

Chesbrough H (2010) Business Model Innovation: Opportunities and Barriers. Long Range Planning 43:354-363

Crunchbase (2021a) What we do. In: about us. https://about.crunchbase.com/about-us/. Zugegriffen: 29. Jan. 2021

Crunchbase (2021b) How we get our data. In: partners. https://about.crunchbase.com/partners/. Zugegriffen: 29. Jan. 2021

Deimel K (2008) Stand der strategischen Planung in kleinen und mittleren Unternehmen (KMU) in der BRD. Z Planung Unternehmenssteuerung 19:281-298

Doraszelski U (2004) Innovations, improvements, and the optimal adoption of new technologies. J Econ Dyn Control 28:1461-1480

Dutta PK, Lach S, Rustichini A (1995) Better late than early: vertical differentiation in the adoption of a new technology. J Econ Manag Strategy 4:563-589

Engelmann N (2018) Virtual Reality Gaming: Potential der Technologie für die Welt der digitalen Spiele. Tectum, Baden-Baden

Fenn J, Raskino M (2008) Mastering the hype cycle: how to choose the right innovation at the right time. Harvard Business Press, Boston

Fenn J, Raskino M, Burton B (2013) Understanding Gartner's hype cycles. Gartner Inc, Stamford 
Fudenberg D, Tirole J (1985) Preemption and rent equalization in the adoption of new technology. Review of Economic Studies 52:383-401

Gartner, Inc. (2004) Hype Cycle for Emerging Technologies, 2004. https://www.gartner.com/en/documents/ 453677/hype-cycle-for-emerging-technologies-2004. Zugegriffen: 5. Juli 2021

Gartner, Inc (2007) Hype Cycle for Emerging Technologies, 2007. https://www.gartner.com/en/documents/ 509710/hype-cycle-for-emerging-technologies-2007. Zugegriffen: 5. Juli 2021

Gartner, Inc (2013) Hype cycle for emerging technologies, 2013. https://www.gartner.com/en/documents/ 2571624/hype-cycle-for-emerging-technologies-2013. Zugegriffen: 5. Juli 2021

Gartner, Inc (2015) Hype cycle for emerging technologies, 2015. https://www.gartner.com/en/documents/ 3100227/hype-cycle-for-emerging-technologies-2015. Zugegriffen: 15. Juli 2021

Gartner, Inc (2016) Hype cycle for emerging technologies, 2016. https://www.gartner.com/en/documents/ 3383817/hype-cycle-for-emerging-technologies-2016. Zugegriffen: 15. Juli 2021

Gartner, Inc (2017) Hype cycle for emerging technologies, 2017. https://www.gartner.com/en/documents/ 3768572/hype-cycle-for-emerging-technologies-2017. Zugegriffen: 15. Juli 2021

Gartner, Inc. (2014) Hype cycle for emerging technologies, 2014. https://www.gartner.com/en/documents/ 2809728/hype-cycle-for-emerging-technologies-2014. Zugegriffen: 15. Juli 2021

Ghoshal S, Nohria N (1993) Horses for Cources: Organizational forms for multinational corporations. MIT Sloan Management Review 34:23-35

Gilbert JT, Birnbaum-More PH (1996) Innovation timing advantages: from economic theory to strategic application. J Eng Technol Manag 12:245-266

van Gisbergen M, Kovacs M, Campos F et al (2019) What we don't know. The effect of realism in virtual reality on experience and behaviour. In: Dieck MC, Jung T (Hrsg) Augmented reality and virtual reality. Springer, Cham, S 45-57

Godefroid P, Kühnle BA (2018) Medientechnologie als unternehmerischer Diversifikationsfaktor. In: Krone J, Pellegrini T (Hrsg) Handbuch Medienökonomie. Springer VS, Wiesbaden, S 1-21

Goldman Sachs Group (2016) Virtual \& Augmented Reality. Understanding the race for the next computing platform

Goodman RA (1973) Environmental knowledge and organizational time horizon: some functions and dysfunctions. Hum Relat 26:215-226

Grand View Research (2021) Virtual reality headset market size, share \& trends analysis report by end device, 2021-2028. https://www.grandviewresearch.com/industry-analysis/virtual-reality-vr-headsetmarket. Zugegriffen: 28. Apr. 2021

Hansen JA (1992) Innovation, firm size, and firm age. Small Bus Econ 4:37-44

Hess T (2019) Digitale Transformation strategisch steuern: Vom Zufallstreffer zum systematischen Vorgehen. Springer, Wiesbaden

Hess T, Matt C, Benlian A, Wiesböck F (2016) Options for Formulating a Digital Transformation Strategy. MIS Quarterly Executive 15:123-139

Hitt MA, Ireland RD, Hoskisson RE (1995) Strategic management: competitiveness and globalization: concepts. West Pub, St. Paul

Hoppe HC (2002) The timing of new technology adoption: theoretical models and empirical evidence. Manch Sch 70:56-76

Hoppe HC, Lehmann-Grube U (2001) Second-mover advantages in dynamic quality competition. J Econ Manag Strategy 10:419-433

Hubert B (2016) Grundlagen des operativen und strategischen Controllings. Springer, Wiesbaden

IABM (2018) Media tech trends. Immersive experiences

IDC (2020a) AR \& VR headsets market share. In: IDC: the premier global market intelligence company. https://www.idc.com/promo/arvr. Zugegriffen: 28. Apr. 2021

IDC (2020b) Prognose zum Absatz von Virtual-Reality- und Augmented-Reality-Brillen weltweit von 2019 bis 2024 (in Millionen Stück). In: Statista. https:/de.statista.com/statistik/daten/studie/539653/ umfrage/prognose-zum-absatz-von-virtual-reality-hardware/. Zugegriffen: 28. Apr. 2021

Institut für Medien- und Kommunikationspolitik (2020) Ranking - Die 50 größten Medienkonzerne 2018. https://www.mediadb.eu/rankings/intl-medienkonzerne-2018.html. Zugegriffen: 10. Dez. 2020

Jensen R (1982) Adoption and diffusion of an innovation of uncertain profitability. J Econ Theory 27:182-193

Jensen R (1992) Innovation adoption and welfare under uncertainty. J Ind Econ 40:173-180

Kind S, Ferdinand J-P, Jetzke T et al (2019) Virtual und Augmented Reality Status quo, Herausforderungen und zukünftige Entwicklungen TA-Vorstudie. Büro für Technikfolgen-Abschätzung beim Deutschen Bundestag, Berlin 
Klingebiel R, Joseph J (2016) Entry timing and innovation strategy in feature phones: entry timing and innovation strategy. Strat Mgmt J 37:1002-1020

Kraus S, Harms R, Schwarz EJ (2006) Strategic planning in smaller enterprises-new empirical findings. Manag Res News 29:334-344

Letts CW, Ryan W, Grossman A (1997) Virtuous capital: what foundations can learn from venture capitalists. Harv Bus Rev 75:36-50

Lieberman MB, Montgomery DB (1988) First-mover advantages. Strat Mgmt J 9:41-58

Loderer C, Waelchli U (2010) Firm age and performance. University Library of Munich, München

Lumpkin GT, Brigham KH (2011) Long-term orientation and intertemporal choice in family firms. Entrepreneursh Theory Pract 35:1149-1169

McCardle KF (1985) Information acquisition and the adoption of new technology. Manage Sci 31: 1372-1389

Mekni M, Lemieux A (2014) Augmented reality: Applications, challenges and future trends. Applied Computational Science 20:205-214

Mezger S, Violani M (2011) Seven basic strategic missteps and how to avoid them. Strategy Leadersh 39:19-26

Miles RE, Snow CC (1978) Organizational strategy, structure, and process. McGraw-Hill, New York

Milgram P, Takemura H, Utsumi A, Kishino F (1995) Augmented reality: a class of displays on the reality-virtuality continuum. In: Proceedings in SPIE. Telemanipulator and telepresence technologies, S 282-292

Müller-Stewens G, Lechner C (2005) Strategisches Management: Wie strategische Initiativen zum Wandel führen (3., aktualisierte Aufl.). Schäffer-Poeschel, Stuttgart

Mütterlein J, Berger B, Hess T (2017) Virtual Reality und die Medienbranche. MW 14:26-31. https://doi. org/10.15358/1613-0669-2017-1-26

Nötzold M (1994) Unternehmensstrategien: Das hierarchische Strategie-Integrationsmodell (SIM) zur Gestaltung erfolgswirksamer Unternehmensstrategien bei Industriebetrieben. Linde, Wien

PwC (2020) German Entertainment and Media Outlook 2020-2024. Fakten, Prognosen und Trends für 13 Segmente der Entertainment- und Medienbranche in Deutschland. PricewaterhouseCoopers,

Reilly G, Souder D, Ranucci R (2016) Time horizon of investments in the resource allocation process: review and framework for next steps. J Manage 42:1169-1194

Reinganum JF (1981) On the diffusion of new technology: a game theoretic approach. Review of Economic Studies 48:395-405

Rogers EM (2003) Diffusion of innovations, 5. Aufl. Free Press, New York

Scholz C (2000) Strategische Organisation: Multiperspektivität und Virtualität (2., überarb. Aufl). Verl. Moderne Industrie, Landsberg/Lech

Schumann M, Hess T, Hagenhoff S (2014) Grundfragen der Medienwirtschaft: eine betriebswirtschaftliche Einführung. Springer Gabler, Berlin

Simon U (2019) Das sind die 100 größten Medienkonzerne der Welt. https:/www.horizont.net/medien/ nachrichten/ranking-das-sind-die-100-groessten-medienkonzerne-der-welt-177843?crefresh=1.

Zugegriffen: 17. Dez. 2020

Slater SF, Mohr JJ (2006) Successful development and commercialization of technological innovation: insights based on strategy type. J Prod Innov Manag 23:26-33

Smith JA (1998) Strategies for start-ups. Long Range Plann 31:857-872

Sołoducho-Pelc L (2015) Planning horizon as a key element of a competitive strategy. J Econ Bus Manag 3:161-166

Stenbacka R, Tombak MM (1994) Strategic timing of adoption of new technologies under uncertainty. Int J Ind Organ 12:387-411

Stonehouse G, Pemberton J (2002) Strategic planning in SMEs-some empirical findings. Manag Decis 40:853-861

Teece DJ (2010) Business Models, Business Strategy and Innovation. Long Range Planning 43:172-194

TrendForce (2020) AR and VR headset shipments worldwide 2020-2025. In: Statista. https://www.statista. com/statistics/653390/worldwide-virtual-and-augmented-reality-headset-shipments/. Zugegriffen: 28. Apr. 2021

Voci D, Karmasin M, Nölleke-Przybylski P et al (2019) What is a media company today? Rethinking theoretical and empirical definitions. Stud Commun Media 8:29-52

Wilczek B (2019) Complexity, uncertainty and change in news organizations. Int J Media Manag 21:88-129

Wohlgenannt I, Simons A, Stieglitz S (2020) Virtual Reality. Business \& Information Systems Engineering 62:455-461 
Zabel C, Telkmann V (2020) The adoption of emerging technology-driven media innovations. A comparative study of the introduction of virtual and augmented reality in the media and manufacturing industries. J Media Bus Stud 18:1-32

Zellweger T (2007) Time Horizon, Costs of Equity Capital, and Generic Investment Strategies of Firms. Fam Bus Rev 20:1-15

Zott C, Amit R, Massa L (2011) The Business Model: Recent Developments and Future Research. Journal of Management 37:1019-1042 